\title{
A VISCOELASTIC COHESIVE LAW FOR RATE AND TEMPERATURE DEPENDENT MIXED MODE DELAMINATION
}

\author{
A.S. Ganapathi ${ }^{*}$, G. Allegri, S. R. Hallett \\ Bristol Composites Institute, University of Bristol, Bristol, United Kingdom \\ * gana.ammasaisengodan@bristol.ac.uk
}

Interlaminar failure of laminated composites is adequately described using bilinear/exponential cohesive zone modelling (CZM) simulations. However, state-of-the-art CZM formulations do not account for the environmental conditions that composite structures encounter in-service. Further enhancements of CZM models are required to account the effects of temperature and strain rate on the delamination behaviour. These effects could be modelled via fitting experimental trends, but such an approach would not provide a comprehensive explanation of the underlying physical mechanisms. In this work, a cohesive zone modelling framework that accounts the effect of loading rate and temperature on the interlaminar failure of laminated composites is presented. The loading and softening part of the traction-separation curve is represented by using the generalized Maxwell model, while the Zhurkov's kinetic theory of failure [1] is employed to describe progressive damage growth. The corresponding rheological elements introduced for a mixed mode-delamination are illustrated in Figure 1(a).

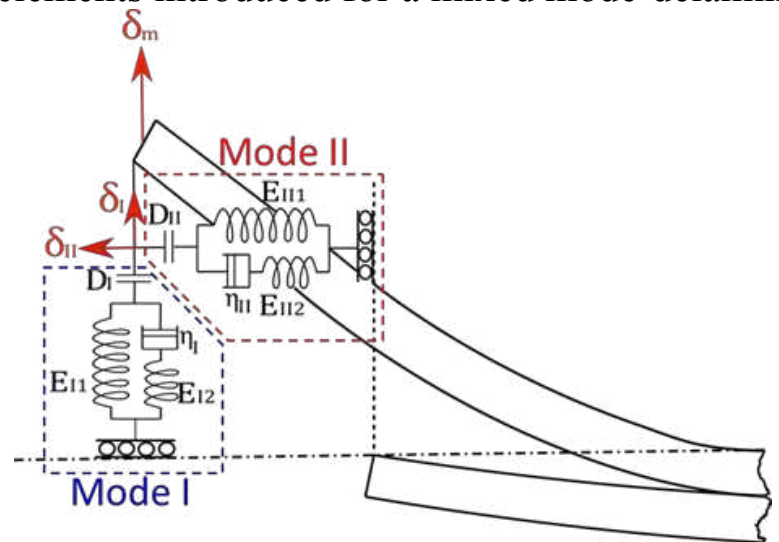

(a)

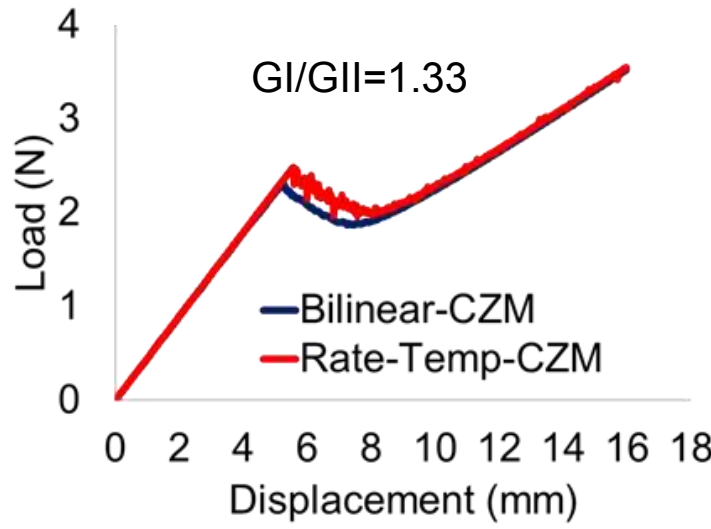

(b)

Figure 1. (a) Rheological representation of the mixed mode delaminations (b) Predictability of the proposed model against the bi-linear law for a fixed ratio mixed mode case [2].

For a given loading rate, the proposed model is able to match the delamination prediction from more commonly used bilinear models calibrated on quasi-static experiments, as shown in Figure 1 (b). However, the new CZM model can also predict the effects of different loading rates and temperature conditions without introducing additional parameters. The energy dissipation due to the viscoelastic response of the cohesive interface is comprehensively described in the current modelling framework. The proposed CZM formulation has been implemented in Abaqus/Explicit as a VUMAT material user subroutine.

\section{References}

[1] S.N. Zhurkov, Kinetic concept of the strength of solids (1984), International Journal of Fracture, 26, :295-307.

[2] P.W. Harper, L. Sun, and S.R. Hallett (2012) A study on the influence of cohesive zone interface element strength parameters on mixed mode behaviour, Composites: Part A, 43, 722-734. 